\section{Choroid Plexus Papilloma with Osseous Metaplasia as a Differential Diagnosis of Calcifying Pseudoneoplasms of the Neuraxis}

We read with special interest the article of Aiken et $\mathrm{al}^{1}$ entitled "Calcifying Pseudoneoplasms of the Neuraxis: CT, MR Imaging, and Histologic Features." The authors reported the imaging and histologic findings of 4 cases of this rare tumor, calcifying pseudoneoplasm of the neuraxis (CAPNON). They suggested that the CAPNON should be considered in the differential diagnosis of intra- and extraaxial calcified masses of the central nervous system. Aiken et al considered the following imaging findings as important in allowing the radiologist to suggest this diagnosis: solid attenuated calcification on CT and a well-defined lesion uniformly hypointense on T1- and T2weighted images, with minimal linear or serpiginous internal enhancement and no edema on MR images. Finally, the authors mentioned that the preoperative diagnosis could be a challenge because there are additional conditions with imaging findings very similar to those of CAPNON.

We report the case of a 34-year-old man with a 9-year history of seizures, who presented with a CT scan showing a heavily calcified mass in the left temporal horn. MR imaging demonstrated a welldefined lesion homogeneously hypointense on T1-, T2-, and susceptibility-weighted MR phase images, with minimal serpiginous inter- nal enhancement and no edema (Fig 1). Although we suggested CAPNON as a possible diagnosis, the histopathologic examination showed matrix calcification interspersed with papillae, lined by cuboidal epithelium, connective tissue, and nervous tissue, associated with foci of trabecular bone, defining the diagnosis of choroid plexus papilloma with ossification areas. The patient presented free of seizures 3 months after the surgery.

We report this case of choroid plexus papilloma with osseous metaplasia, which is a very rare lesion, ${ }^{2-4}$ because of the similarity of the imaging findings to those of CAPNON. Previous reports of ossifying papilloma showed that while the calcification has been documented radiologically in $4 \%-25 \%$ of the cases with choroid plexus papilloma, the metaplasia ossea in this tumor is very rare and has been reported in only some cases in the literature. Imaging features of most reported cases ${ }^{2,3}$ were strikingly similar to those in our case, a calcified mass on CT and the uniform hypointensity on both T1- and T2weighted imaging, with minimal linear internal enhancement and no edema.

Finally, like Aiken et al, ${ }^{1}$ who suggested that choroid plexus tumors be included in the differential diagnosis of CAPNON, we emphasize that specifically the ossifying papilloma should be considered in the differential diagnosis of a calcified mass of the lateral ventricle,
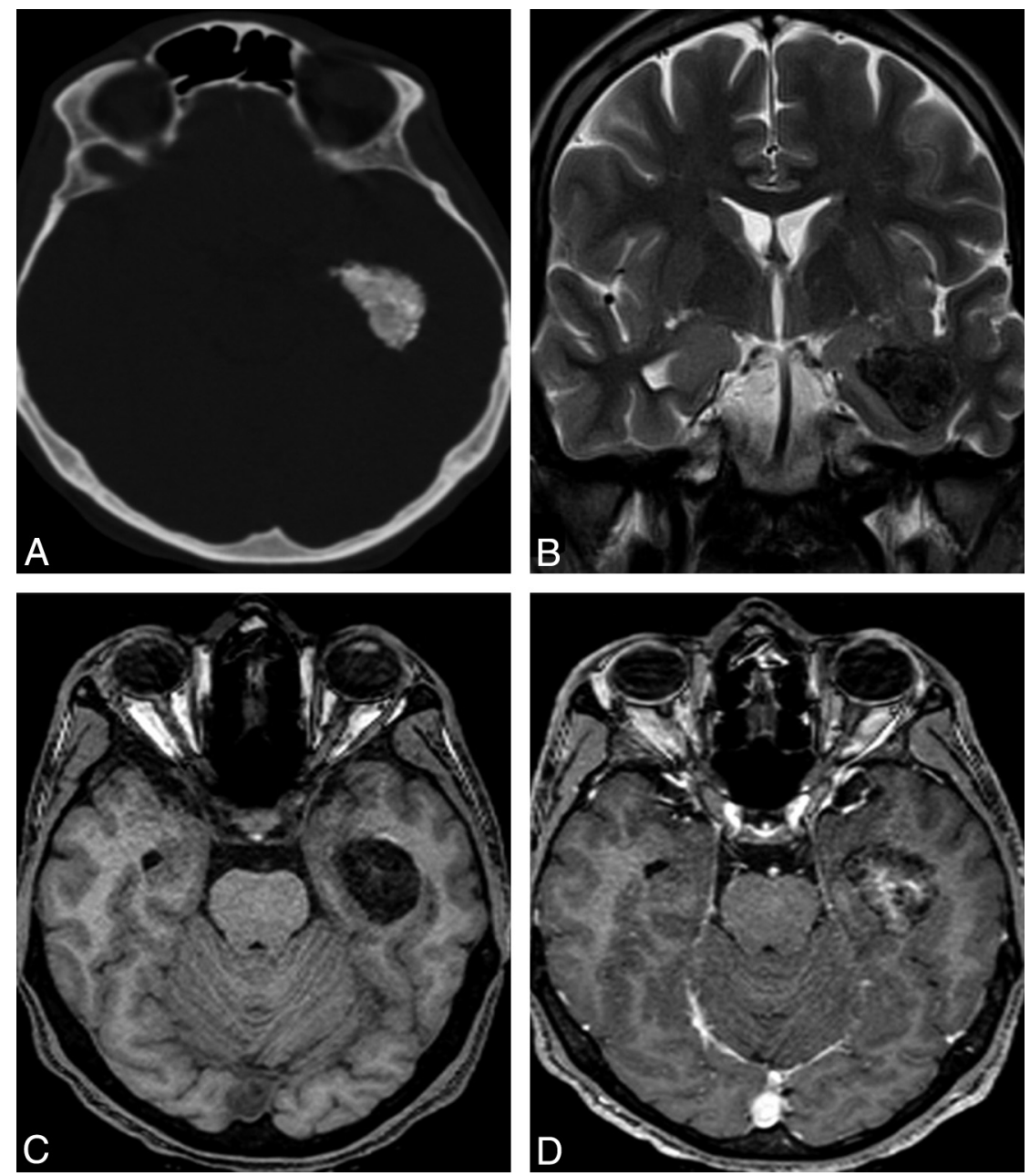

Fig 1. $A$, Noncontrast CT scan shows a heavily calcified mass in the left temporal horn. $B$, Coronal T2-weighted MR image shows a uniform hypointense mass and no edema. $C$, Axial T1-weighted MR image demonstrates a well-defined homogeneously hypointense lesion centered in the left temporal horn. $D$, Axial T1-weighted MR image after gadolinium administration reveals minimal serpiginous internal enhancement. 
such as a calcified meningioma, oligodendroglioma, ependymoma, giant cell astrocytoma, and granuloma. ${ }^{2}$

\section{References}

1. Aiken AH, Akgun H, Tihan T, et al. Calcifying pseudoneoplasms of the neuraxis: CT, MR imaging, and histologic features. AJNR Am J Neuroradiol 2009;30:1256-60

2. Kocaeli H, Yilmazlar S, Abas F, et al. Total ossification of choroid plexus papilloma mimicking calcified petrous bone pathology. Pediatr Neurosurg 2007; 43:67-71

3. Cardozo J, Cepeda F, Quintero M, et al. Choroid plexus papilloma containing bone. Acta Neuropathol 1985;68:83-85
4. Corcoran GM, Frazier SR, Prayson RA. Choroid plexus papilloma with osseous and adipose metaplasia. Ann Diagn Pathol 2001;5:43-47

D.B. Pereira

E.L. Gasparetto

Department of Radiology

J. Marcondes de Souza

Department of Surgery

L. Chimelli

Department of Pathology

University Federal of Rio de Janeiro

Rio de Janeiro, Brazil

DOI 10.3174/ajnr.A2096 